\title{
Fungal Growth Rate and the Formation of Ethylene in Soil
}

\author{
By J. M. LYNCH AND S. H. T. HARPER \\ Agricultural Research Council, Letcombe Laboratory, Wantage OXI2 $9 J T$
}

(Received 3I May 1974)

\begin{abstract}
SUMMARY
The rates of ethylene production per unit mass of Mucor hiemalis are highest at low specific growth rates. Thus this fungus probably produces more ethylene in the soil, where growth rates are low, than would have been predicted by laboratory batch culture, where growth rates are high. The rate of utilization of energyyielding substrate by Mucor hiemalis increases logarithmically with growth rate. This contrasts with the data obtained for Penicillium digitatum and Aspergillus nidulans; it is discussed in terms of the success of Mucor hiemalis as a primary saprophytic sugar fungus.
\end{abstract}

\section{INTRODUCTION}

Microbial growth rates in the soil are governed partly by the availability of energy-yielding substrates. The formation of microbial products is controlled by both growth rates of the organisms and the availability of the transformation substrates which are used to form specific products. We have reported previously the effects of substrates on the formation of a plant growth regulator, ethylene, in soil and in pure cultures of the common soil fungus, Mucor hiemalis (Lynch, 1972; Lynch \& Harper, 1974), where glucose is the energy-yielding substrate and methionine is the transformation substrate. At least part of the process of ethylene formation is extracellular (Lynch, 1974a). A problem in extrapolating from laboratory studies to the field is that growth rates of soil micro-organisms in laboratory culture, where there is usually an ample supply of substrates, are much higher than those in the field. Growth rates of filamentous fungi in laboratory flask cultures are often about $0.2 \mathrm{~h}^{-1}$, whereas estimates of microbial growth rates in the soil have ranged from $0.0006 \mathrm{~h}^{-1}$ (Babiuk \& Paul, 1970) to $0.04 \mathrm{~h}^{-1}$ (Gray \& Williams, I97I). It therefore seemed to be important to control availability of substrate and hence growth rate in laboratory cultures, when predicting the behaviour of $M$. hiemalis in soil.

Pirt (1965) discussed the effect of maintenance energy, i.e. energy required for functions other than the production of new cell material, on the growth yield of bacteria. Studies of maintenance energy requirements have now been extended to the fungi (Righelato, Trinci, Pirt \& Peat, 1968; Carter, Bull, Pirt \& Rowley, 197I). The true growth yield of a microorganism, $Y_{g}$, does not allow for substrate consumption in cell maintenance, whereas the observed growth yield includes this. The metabolic quotient or specific rate of energy substrate utilization, $q$ (g material metabolized/g dry wt organisms/h), can be described as follows:

$$
q=\mu / Y_{g}+m
$$

where $\mu$ is specific growth rate and $m$ is the maintenance coefficient. Hence if $q$ is plotted against $\mu$, a linear relationship of slope $\mathrm{I} / Y_{g}$ and intercept $m$ is predicted. This occurs in the utilization of glucose by Penicillium chrysogenum (Righelato et al. 1968) and by Aspergillus nidulans (Carter et al. I97I). However, the linear relationship applies for A. nidulans only at growth rates below $0.06 \mathrm{~h}^{-1}$. 


\section{METHODS}

Inoculum of Mucor hiemalis ATCC26035, isolated from a heavy clay soil, was prepared as described previously (Lynch \& Harper, 1974). Glucose-limited chemostat cultures were grown on mineral salts medium (Lynch \& Harper, 1974) with glucose at $9 \mathrm{~g} / 1$ and DLmethionine at I $\mathrm{g} / \mathrm{l}$, at $25^{\circ} \mathrm{C}, \mathrm{pH} 6 \cdot 0$, and dissolved oxygen tension always greater than $85 \%$ of air saturation. Ethylene, oxygen, carbon dioxide, methionine and glucose were assayed as described previously but an automatic procedure was employed for gas sampling (Lynch, I974 b). The specific metabolic procedure was employed for gas sampling (Lynch, 1974b). The specific metabolic quotients for glucose and oxygen were calculated according to Carter et al. (197I). Extracellular carbon in the spent medium was estimated by adding with swirling $0.2 \mathrm{M}$-potassium dichromate $(10 \mathrm{ml})$ in concentrated sulphuric acid $(20 \mathrm{ml})$ to a sample of the medium $(2 \mathrm{ml})$ in a $250 \mathrm{ml}$ conical flask. After cooling for $30 \mathrm{~min}$, de-ionized water $(70 \mathrm{ml})$ was added and the colour of the solution measured spectrophotometrically at $625 \mathrm{~nm}$. This was compared with glucose standards in the range I to $5 \mathrm{mg}$ carbon/2 ml. It was assumed that the cellular carbon content of the fungus was $50 \%$ of its dry weight.

\section{RESULTS}

\section{Ethylene production}

There was a great increase in ethylene production per unit weight of organism per unit time as the specific growth rate was decreased below $0.04 \mathrm{~h}^{-1}$ (Fig. I). The maximum specific growth rate observed in batch cultures was $0.21 \mathrm{~h}^{-1}$, but in continuous cultures the organism washed out at dilution rates in excess of $0.08 \mathrm{~h}^{-1}$. Wash-out at dilution rates substantially below that required to give maximum specific growth rate has been observed previously for moulds (Righelato et al. 1968; Carter et al. 1971).

In the chemostat at a specific growth rate of $0.018 \mathrm{~h}^{-1}, M$. hiemalis produced ethylene at a rate per unit mass of organism which is about 1000 times greater than that in sealed shaken flasks. Although reduced oxygen levels are partially responsible for the much lower concentrations of ethylene observed in the flasks (Lynch \& Harper, 1974), the high growth rate is probably the major factor responsible.

Increased ethylene formation was the result of an increased conversion of methionine to ethylene and not to an increase in methionine uptake, which was about $90 \%$ irrespective of growth rate.

\section{Specific rate of glucose utilization}

For $M$. hiemalis where glucose is the energy substrate, the relationship between the utilization of energy substrate and the specific growth rate is not linear but can instead be described by a logarithmic curve which extrapolates at $\mu=0$ to give $m=0.04 \pm 0.0 \mathrm{I} \mathrm{h}^{-1}$. This discrepancy from the predicted linear relationship might be caused by a false assumption that $m$ is independent of $\mu$. The substrate utilization curve approximately mirrors that for ethylene production at increasing $\mu$ values.

The specific rates of energy utilization by $M$. hiemalis were much higher than those obtained by other workers for $P$. chrysogenum and $A$. nidulans (Table I). The maintenance energy also appeared to be higher for $M$. hiemalis, but this difference may merely reflect the difficulty in extrapolating the curves to obtain these values and therefore may not be significant.

\section{Respiration}

The specific rate of oxygen utilization increased with growth rate (Table 2), as has been found previously for moulds, but the values were considerably greater than those observed 


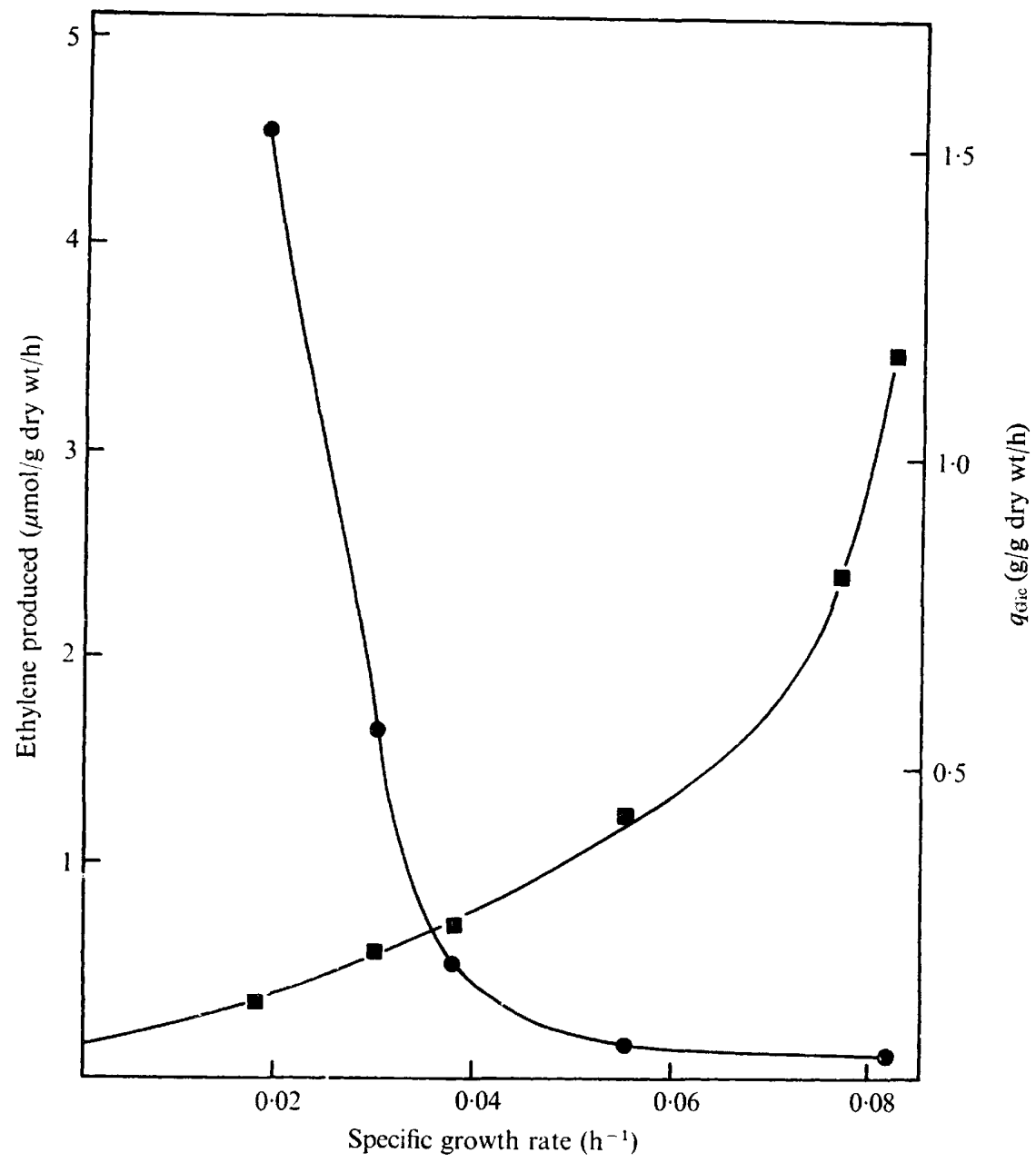

Fig. I. Ethylene production and energy substrate (glucose) utilization at different specific growth rates by chemostat cultures of Mucor hiemalis.

, Ethylene; $\mathbf{\square}, q_{\mathrm{Glc}}$.

for $P$. chrysogenum and $A$. nidulans at the corresponding growth rates, with a greater differential at higher growth rates. This seems to be consistent with the values of $q_{\mathrm{Glc}}$. The specific rate of carbon dioxide production increased similarly to give respiratory quotients (R.Q.) just above I; R.Q. increased with growth rate. For $P$. chrysogenum, R.Q. was always just below I.O whereas for $A$. nidulans it was always about I.0; in both cases R.Q. increased with growth rate.

\section{Carbon balance}

The high specific rate of glucose utilization at high growth rates suggested that the increased carbon utilized per unit mass was secreted as extracellular products. It was shown that there was an increase in extracellular products with growth rate (Table 3), but with increased growth rate progressively less of the carbon utilized could be accounted for. Whilst the analysis of carbon present is accurate to within a few per cent of the total carbon, the procedure used did not include estimation of volatile products. Unfortunately, the samples could not be analysed immediately and had to be deep-frozen and thawed twice before analysis, which would accentuate any loss of volatile carbon products in addition to those lost as a 
Table 1. Maintenance energies and specific rates of energy substrate utilization at different specific growth rates of fungi

\begin{tabular}{|c|c|c|c|c|}
\hline \multirow[b]{2}{*}{ Fungus } & \multirow{2}{*}{$\begin{array}{l}\text { Maintenance } \\
\text { coefficient, } m \\
\left(\mathrm{~h}^{-1}\right)\end{array}$} & \multicolumn{2}{|c|}{$q_{\text {Gle }}(\mathrm{g} / \mathrm{g}$ dry wt $/ \mathrm{h})$} & \multirow[b]{2}{*}{ Reference } \\
\hline & & $\mu=0.02 \mathrm{~h}^{-1}$ & $\mu=0.08 \mathrm{~h}^{-1}$ & \\
\hline Penicillium chrysogenum & 0.02 & 0.06 & 0.19 & Righelato et al. (1968) \\
\hline Aspergillus nidulans & 0.02 & 0.05 & 0.20 & Carter et al. (1971) \\
\hline Mucor hiemalis & 0.04 & 0.12 & 0.99 & This paper \\
\hline
\end{tabular}

Table 2. Respiration of Mucor hiemalis at different specific growth rates

$\begin{array}{cccc}\text { Specific growth } & \begin{array}{c}\text { Specific rate of } \\ \text { oxygen utilization } \\ \text { rate, } \mu(\mathrm{g} / \mathrm{g} \text { dry } \mathrm{wt} / \mathrm{h})\end{array} & \begin{array}{c}\text { Specific rate of } \\ \text { carbon dioxide } \\ \text { production } \\ (\mathrm{g} / \mathrm{g} \text { dry } w \mathrm{t} / \mathrm{h})\end{array} & \begin{array}{r}\text { Respiratory } \\ \text { quotient, R.Q. }\end{array} \\ 0.018 & \mathrm{I} 8 & 2 \cdot 23 & \mathrm{I} \cdot 25 \\ 0.038 & 3.55 & 4.65 & \mathrm{I} \cdot 3 \mathrm{I} \\ 0.082 & 6.47 & 8 \cdot 95 & \mathrm{I} \cdot 38\end{array}$

Table 3. Fate of carbon fed to Mucor hiemalis at different specific growthrates

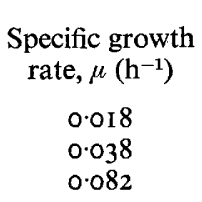

Specific growth

0.018
0.038
0.082

Carbon

Carbon feed was glucose $(9 \mathrm{~g} / \mathrm{l})$, which was all utilized at all growth rates, and DL-methionine (I $\mathrm{g} / \mathrm{l})$, of which $90 \%$ was utilized at all growth rates.

result of the continuous gas flow in the fermenter itself. It therefore seems reasonable to assume that a proportion of the unaccounted for carbon was lost in volatile products. This would be consistent with the observations of others who have shown that the production of volatile fatty acids and ethanol by yeasts, bacteria and fungi increases with growth rate (Maxon \& Johnston, I953; Pirt, 1957; Pirt \& Callow, 1960).

For batch cultures in sealed shaken flasks, where the organism grows at its maximum specific growth rate $\left(0.2 \mathrm{I} \mathrm{h}^{-1}\right)$ but where oxygen is limiting, $3 \mathrm{I} \%$ of the carbon utilized resulted in cellular material and $14 \%$ resulted in carbon dioxide, with $55 \%$ unaccounted for, presumably as extracellular products.

\section{Morphology}

It is well known that the genus Mucor exhibits mould-yeast dimorphism (Bartnicki-Garcia, I963). It is also known that growth rate influences the morphology of $P$. chrysogenum (Righelato et al. I968) and a soil bacterium (Luscombe \& Gray, 197I). The characteristic growth form of $M$. hiemalis growing in submerged liquid culture (Fig. 2) was observed at all growth rates studied in the chemostat culture and in flask culture. The yeast form of the organism was never observed in chemostat culture although it was occasionally noticed in flask cultures; however the occurrence of the yeast form did not appear to be related to any particular physical or chemical conditions. The organism always grew in a filamentous form in the chemostat, although pellets were sometimes observed in flasks when an inoculum giving less than $10^{4}$ spores $/ \mathrm{ml}$ medium was used. 


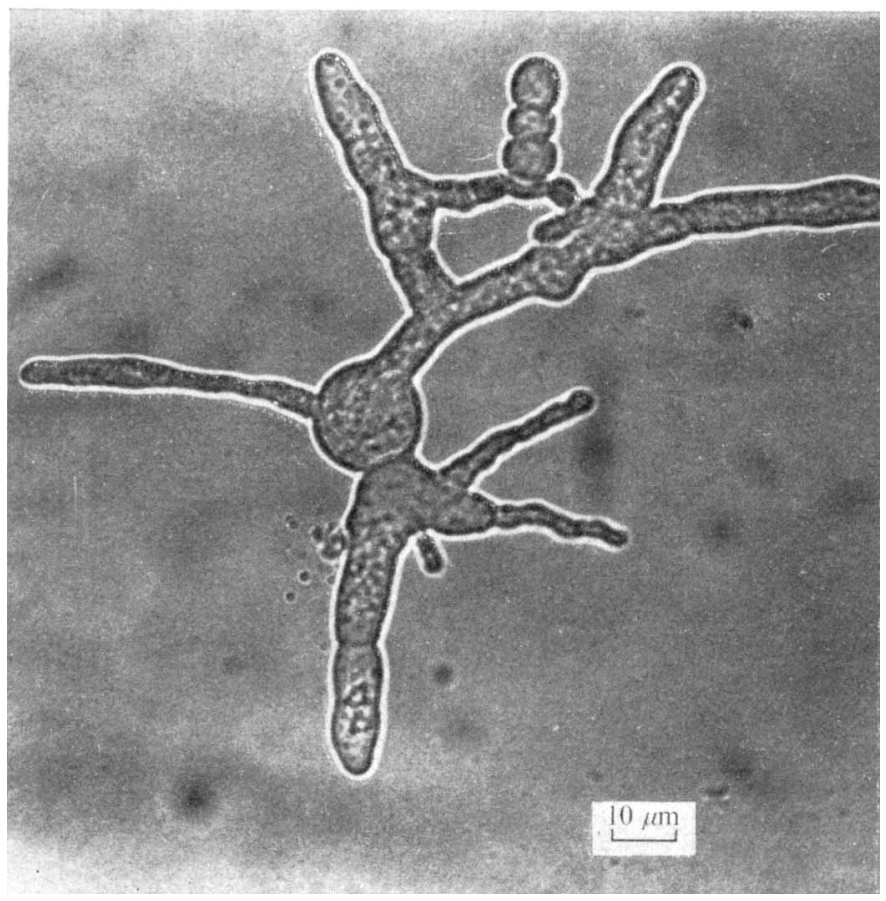

Fig. 2. Mucor hiemalis growing in"submerged liquid culture.

\section{DISCUSSION}

The results show clearly that at growth rates which might be expected in soils, ethylene production is likely to be greater than would have been predicted by conventional laboratory batch culture techniques (Lynch, 1972; Lynch \& Harper, 1974). They also emphasize the point that growth rate should be considered when extrapolating microbial behaviour in laboratory culture to field situations. Furthermore, it is evident from the work of others (Tempest \& Herbert, 1965; Carter \& Bull, 1969) that growth rate has a considerable effect on the enzyme activity of fungi, and the present results are a further demonstration of such changes.

Mucor hiemalis seems to be important in ethylene production in soil because it is a pioneer colonizer of fresh plant tissue substrates, where it can get substrates for growth and ethylene formation (Lynch, 1972), i.e. it succeeds as a primary saprophytic sugar fungus as described by Garrett (1970). The present results showing that $M$. hiemalis has a high rate of utilization of energy-yielding substrate compared with $P$. chrysogenum and $A$. nidulans may partly explain why it is successful as a primary colonizer. The production of large amounts of extracellular products by $M$. hiemalis might provide substrates for secondary colonizers in soil.

\section{REFERENCES}

Babiuk, L. A. \& PaUl, E. A. (1970). The use of fluorescein isothiocyanate in the determination of the bacterial biomass of grassland soil. Canadian Journal of Microbiology 16, 57-62.

BARTNICKI-GarCía, S. (I963). Mold-yeast dimorphism of Mucor. Bacteriological Reviews 27, 293-304.

Carter, B. L. A. \& Bull, A. T. (I969). Studies of fungal growth and intermediary carbon metabolism under steady and non-steady state conditions. Biotechnology and Bioengineering $\mathbf{1}$, 785-804. 7 
CARTer, B. L. A., Bull, A. T., PiRT, S. J. \& Rowley, B. I. (1971). Relationship between energy substrate utilization and specific growth rate in Aspergillus nidulans. Journal of Bacteriology 108, 309-313.

Garrett, S. D. (1970). Pathogenic root-infecting fungi. Cambridge University Press.

Gray, T. R. G. \& Williams, S. T. (I97I). Microbial productivity in soils. In Microbes and Biological Productivity, pp. 255-286. Edited by D. E. Hughes and A. H. Rose. Cambridge University Press.

LusCOMBE, B. M. \& GRAY, T. R. G. (197I). Effect of varying growth rates on the morphology of Arthrobacter. Journal of General Microbiology 69, 433-434.

LYNCH, J. M. (1972). Identification of substrates and isolation of micro-organisms responsible for ethylene production in the soil. Nature, London 240, 45-46.

LYNCH, J. M. (1974a). Mode of ethylene formation by Mucor hiemalis. Journal of General Microbiology 83, 407-4II.

LYNCH, J. M. (1974b). Automated sampling of gases from microbial cultures. Journal of Applied Bacteriology 37 (in the Press).

LYNCH, J. M. \& HARPER, S. H. T. (1974). Ethylene formation by a soil fungus. Journal of General Microbiology 80, I 87-195.

MAXON, W. D. \& Johnston, M. J. (I953). Aeration studies on propagation of baker's yeast. Industrial and Engineering Chemistry 45, 2554-2560.

PIRT, S. J. (1957). The oxygen requirement of growing cultures of an Aerobacter species determined by means of the continuous culture technique. Journal of General Microbiology 16, 59-75.

PIRT, S. J. (1965). The maintenance energy of bacteria in growing cultures. Proceedings of the Royal Society B I63, 224-23I.

PIRT, S. J. \& Callow, D. S. (1960). Studies on the growth of Penicillium chrysogenum in continuous flow culture with reference to penicillin production. Journal of Applied Bacteriology 23, 87-98.

Righelato, R. C., Trinci, A. P. J., Pirt, S. J. \& Peat, A. (I968). The influence of maintenance energy and growth rate on the metabolic activity, morphology and conidiation of Penicillium chrysogenum. Journal of General Microbiology 50, 399-412.

Tempest, D. W. \& HerberT, D. T. (1965). Effect of dilution rate and growth-limiting substrate on the metabolic activity of Torula utilis cultures. Journal of General Microbiology 4r, I43-150 